RNA INTERFERENCE

\section{Nuts and bolts}

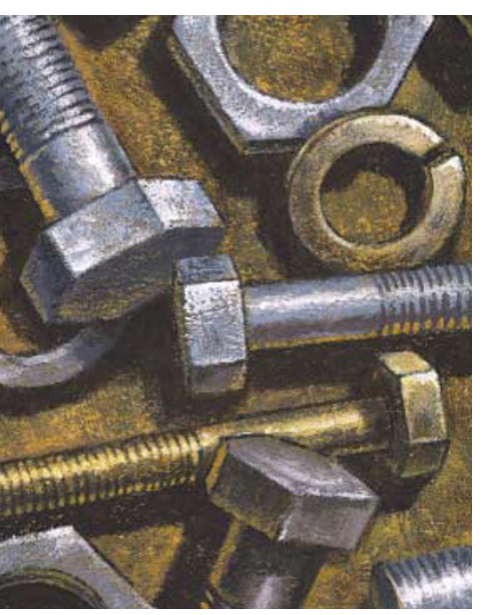

Although rapid progress is being made in understanding the key steps in the RNA interference (RNAi) pathway, it is clear that many of the 'nuts and bolts' of the silencing machinery are still unknown. Craig Mello and colleagues now report, in Current Biology, the characterization of the Caenorhabditis elegans protein RDE-3, and propose a role for this new member of the polymerase- $\beta$ nucleotidyltransferase superfamily in RNAi.

Mello and co-workers had previously genetically mapped the $r d e-3$ locus in an RNAi-deficient strain. Further mapping and examining the candidate genes within the mapped region for mutations led them to the K04F10.6 gene - rescue experiments confirmed that K04F10.6 was indeed $r d e$-3. Three presumed $r d e-3$ loss-of-function mutants showed similar levels of resistance to RNAi when they were injected with double stranded (ds)RNA. The mutants also failed to accumulate small interfering (si)RNA, which implies that RDE-3 functions upstream of siRNA accumulation during RNAi.

RDE-3 contains a conserved nucleotidyltransferase domain, NTP transferase-2, with two conserved features - a helical turn that contains a Gly-Ser motif and an aspartic-acid triad motif. Two rde-3-null alleles encode a mutation in each of these motifs, which implies that the polymerase activity of RDE-3 is likely to be important for its function.
So, how does RDE-3 function in RNAi? Although the authors can't answer this question just yet, they do suggest several interesting possibilities. They propose that RDE-3 might function as a polyadenylation (poly $(A))$ polymerase and that, in the absence of functional RDE-3, aberrant transcripts with short poly(A) tails accumulate - these could compete for limiting factors that are required for efficient RNAi.

Alternatively, Mello and colleagues suggest that RDE- 3 could be a direct component of the RNAi pathway, which is required for the amplification of the RNAi response that is induced by small amounts of dsRNA. The initially produced, lowabundance, primary siRNAs trigger the first round of cleavage of the target mRNA. RDE-3 is then proposed to polyadenylate the cleavage product,

\section{SIGNAL TRANSDUCTION}

\section{A stabilizing modification}

Signal transducer and activator of transcription (STAT) proteins are latent in the cytoplasm until extracellular signals - such as cytokines or growth factors induce their activation, dimerization and translocation to the nucleus. Here, the STAT dimers bind to DNA and promote the transcription of various genes. In response to the extracellular signals, STATs are phosphorylated and this post-translational modification is thought to be necessary for STAT activation. However, as unphosphorylated STATs can still dimerize and induce transcription, Chin and colleagues investigated whether other modifications might be important for STAT activation, and they report their results in Science.

Proteins of the cAMP-response-elementbinding protein (CREB)-binding protein (CBP)/p300 family have intrinsic histoneacetylase activity, and they are known to associate with various STATs and promote STAT-mediated transcription. So, the authors looked at whether STATs can be acetylated, and found that STAT3 acetylation was induced by treating cells with extracellular signalling proteins. In addition, they showed that this acetylation was increased by transfecting cells with $\mathrm{CBP}$ or $\mathrm{p} 300$, and that neither STAT3 phosphorylation, nor the activity of the Src-homology-2 domain of STAT3, were required for this modification. A broad inhibitor of histone deacetylases (HDACs) increased STAT3 acetylation in response to $\mathrm{p} 300$ transfection or cytokine treatment, and Chin and co-workers found that STAT3 deacetylation could be mediated by type-I HDACs. It therefore seems that p300 and type-I HDACs, which are present in most cell types, might control cytokineinduced STAT3 acetylation/deacetylation.

Next, the authors showed that Lys 685 of STAT3, which is conserved in several STATs from various species, was acetylated by $\mathrm{p} 300$, and they investigated the effect of this modification on STAT3 activity. They showed that the dimerization of wild-type STAT3 was stabilized by cytokine treatment and that, by contrast, no dimerization could be detected for Lys685Arg-mutant STAT3 proteins after cytokine treatment, even though these mutant proteins were phosphorylated. Lys685 acetylation therefore seems to be important for STAT3 dimerization. Furthermore, the authors showed that cytokine treatment could induce the formation of complexes between DNA and wild-type STAT3, but not DNA and Lys685Arg STAT3.

In the final part of their study, Chin and colleagues looked at the effect of STAT3 acetylation on cell growth in vivo, as STAT3 regulates several cell-growth- and cellsurvival-related genes. In a cell line that lacks STAT3, the expression level of three such genes was low, with or without cytokine treatment, but, after cytokine treatment, cells that had been transfected with wildtype STAT3 - but not Lys685Arg STAT3 showed increased expression of these genes. They also showed that wild-type, but not Lys685Arg, STAT3 could promote cell-cycle progression and cell growth in response to cytokines.

So, this work indicates that STAT acetylation is necessary for the stable dimerization of STATs and for them to activate transcription. Furthermore, as $\mathrm{CBP} / \mathrm{p} 300$ is known to associate with various STATs, “....it is possible that all STAT family members are tightly regulated by the acetylation and deacetylation cycle."

\section{Rachel Smallridge}

\section{(i) References and links}

ORIGINAL RESEARCH PAPER Yuan, Z.-L. et al. Stat3 dimerization regulated by reversible acetylation of a single lysine residue. Science 307, 269-273 (2005)

FURTHER READING O'Shea, J. J. et al. Cell signaling: Stat acetylation - a key facet of cytokine signaling? Science $\mathbf{3 0 7}$ 217-218 (2005)

WEB SITE

Y. Eugene Chin's laboratory:

http://www.brown.edu/Divisions/Medical_School/andera/pe rson.php?id=299\&returnURL=\%2FDivisions\%2FMedical_Sc hool\%2Fandera\%2Ffaclist.php\%3Ffirst\%3DC 